\title{
Interstitial deletion of $6 q 25.2-q 25.3:$ a novel microdeletion syndrome associated with microcephaly, developmental delay, dysmorphic features and hearing loss
}

\author{
Sandesh Chakravarthy Sreenath Nagamani ${ }^{1}$, Ayelet Erez ${ }^{1}$, Christine Eng ${ }^{1}$, Zhishuo Ou ${ }^{1}$, \\ Craig Chinault ${ }^{1}$, Laura Workman ${ }^{2}$, James Coldwell ${ }^{3}$, Pawel Stankiewicz ${ }^{1}$, Ankita Patel ${ }^{1}$, \\ James R Lupski ${ }^{1,4,5}$ and Sau Wai Cheung ${ }^{\star, 1}$
}

\author{
${ }^{1}$ Department of Molecular and Human Genetics, Baylor College of Medicine, Houston, TX, USA; ${ }^{2}$ Sutter Medical \\ Center, Sacramento, CA, USA; ${ }^{3}$ Children's Medical Center, Tulsa, OK, USA; ${ }^{4}$ Department of Pediatrics, Baylor College \\ of Medicine, Houston, TX, USA; ${ }^{5}$ Texas Children's Hospital, Houston, TX, USA
}

Interstitial deletions of $\mathbf{6 q}$ are rare. We report a detailed clinical and molecular characterization of four patients with interstitial deletion involving 6 q25. All of our patients presented with microcephaly, developmental delay, dysmorphic features and hearing loss, whereas two of them had agenesis of the corpus callosum. We determined the size, extent and genomic content of the deletions using high-density array-comparative genomic hybridization (a-CGH), and found that a common segment spanning $3.52 \mathrm{Mb}$ within the 6q25.2-q25.3 region was deleted in all four cases. We hypothesize that a subset of genes in the commonly deleted region are dosage sensitive and that haploinsufficieny of these genes impairs normal development of the brain and hearing.

European Journal of Human Genetics (2009) 17, 573-581; doi:10.1038/ejhg.2008.220; published online 26 November 2008

Keywords: $6 \mathrm{q}$ deletion; hearing loss; microcephaly, developmental delay; agenesis of the corpus callosum; array-CGH

\section{Introduction}

Since the first reported case in $1975,{ }^{1}$ over 65 cases of deletions involving the long arm of chromosome 6 have been reported. ${ }^{2-7}$ Though the clinical phenotype is quite variable, Hopkin et $a l^{2}$ attempted to correlate the phenotype with genotype, by dividing patients into three groups. Those in Group A with $\operatorname{del}(6)(q 11 q 16)$ had a high incidence of hernias, up-slanting palpebral fissures, thin

${ }^{*}$ Correspondence: Dr SW Cheung, Cytogenetics Laboratory, Department of Molecular and Human Genetics, Baylor College of Medicine, 1 Baylor Plaza, NAB 2015, Houston TX 77030, USA. Tel: + 1713798 6555;

Fax: + 1713798 3157; E-mail: scheung@bcm.tmc.edu

Received 3 July 2008; revised 26 September 2008; accepted 10 October 2008; published online 26 November 2008 lips, a lower frequency of microcephaly, micrognathia and heart malformations. Patients categorized to Group B with del(6)(q15q25) had intrauterine growth retardation, abnormal respiration, hypertelorism and upper limb malformations, whereas those in group C with $\operatorname{del}(6)(\mathrm{q} 25)$ presented with retinal abnormalities, cleft palate and genital hypoplasia. However, there was significant overlap of features among the groups and some features such as developmental delay (100\%), ear anomalies (90\%), hypotonia $(82 \%)$ and postnatal growth retardation (68\%) were common to all groups. Hearing loss has been reported only in a minority of publications. ${ }^{1,2,6,8,9}$

Most of the reported deletions were identified and characterized based on routine karyotyping with no further attempt to delineate the breakpoints. We determined the 
size, extent and genomic content of four cases involving interstitial deletion of $6 \mathrm{q} 25$. All four deletions have a common deleted segment in 6q25.2-q25.3 with the smallest region of overlap (SRO) of $3.52 \mathrm{Mb}$. All of the patients presented with microcephaly, developmental delay, dysmorphic features and hearing loss. We hypothesize that a subset of genes deleted in the SRO are dosage sensitive and are important for CNS and hearing development.

\section{Clinical reports \\ Patient 1}

The proband is a male child born to nonconsanguineous Hispanic parents. Family history was negative for known chromosomal anomalies or birth defects. Pregnancy was uncomplicated and delivery was by Cesarean section that was performed for fetal distress. Birth weight was $3.6 \mathrm{~kg}$ (25th centile), whereas the length and FOC are not available. Apgar scores were 7 and 9 at 1 and $5 \mathrm{~min}$, respectively. The perinatal period was characterized by feeding difficulties. At the 6-month examination, his motor development was that of a 3-month old. A peripheral G-banded karyotyping performed at 6 months was normal. When examined by a genetics consultant at 33 months of age, his FOC $(46.2 \mathrm{~cm})$ was at the 2 nd centile (z-score -2.0$)$, whereas the height $(89.2 \mathrm{~cm})$ and weight $(13.1 \mathrm{~kg})$ were at the 9 th and $27 \mathrm{th}$ centile, respectively. Examination was remarkable for plagiocephaly, downslanting palpebral fissures, epicanthic folds, midface hypoplasia, low set ears, long philtrum and micrognathia (Figure 1a). He had global developmental delay; rolled over at 1 year, walked at 23 months and had only two words at 33 months. Audiology evaluation showed severe sensorineural hearing loss on the right. Brain imaging could not be performed.

\section{Patient 2}

This female child was born at 37 weeks of gestation to nonconsanguineous Caucasian parents. The mother had an abnormal triple screen and underwent amniocentesis that revealed a normal female chromosome constitution, $46, X X$. Growth parameters at birth were remarkable for weight of $2.2 \mathrm{~kg}$ ( 0.3 centile, $z$-score -2.8$)$ and FOC of $31 \mathrm{~cm}$ ( 0.1 centile, $z$-score -3.9$)$, whereas length $48 \mathrm{~cm}$ (5th centile) was normal. Soon after birth, she had respiratory compromise requiring ventilation with the aid of bag and mask. The perinatal period was also complicated by subarachnoid hemorrhage that was managed medically without any surgical intervention. The proband had feeding difficulties until 3 months. Her medical problems also included two episodes of febrile seizures and hypotonia. At the 9-month evaluation, she was noted to have a bulbous nose, thin upper lip and a pit on the right helix (Figure 1b). Audiology evaluation revealed bilateral moderate-to-severe sensorineural hearing loss. MRI of the brain revealed plagiocephaly and no structural abnormalities of the brain. CT scan of the temporal bones showed normal cochlear structure. (Figure 2a) Ophthalmological

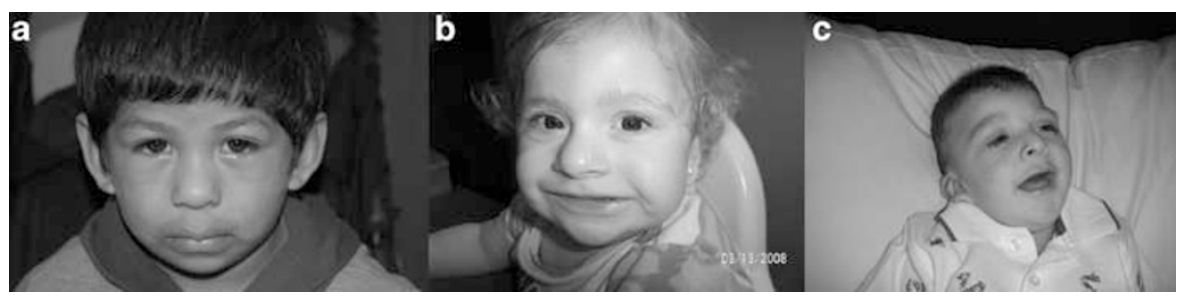

Figure 1 Photographs of patients 1 (a), 2 (b) and 4 (c) showing facial characteristics. Note that hypertelorism, posteriorly rotated auricles, broad nasal root and midface hypoplasia are common to all. In addition, patient 1 has epicanthic folds, down-slanting palpebral fissures and retrognathia; patient 2 has a bulbous nose and thin upper lip, whereas patient 4 has malformed, low-set auricles and a thin upper lip.

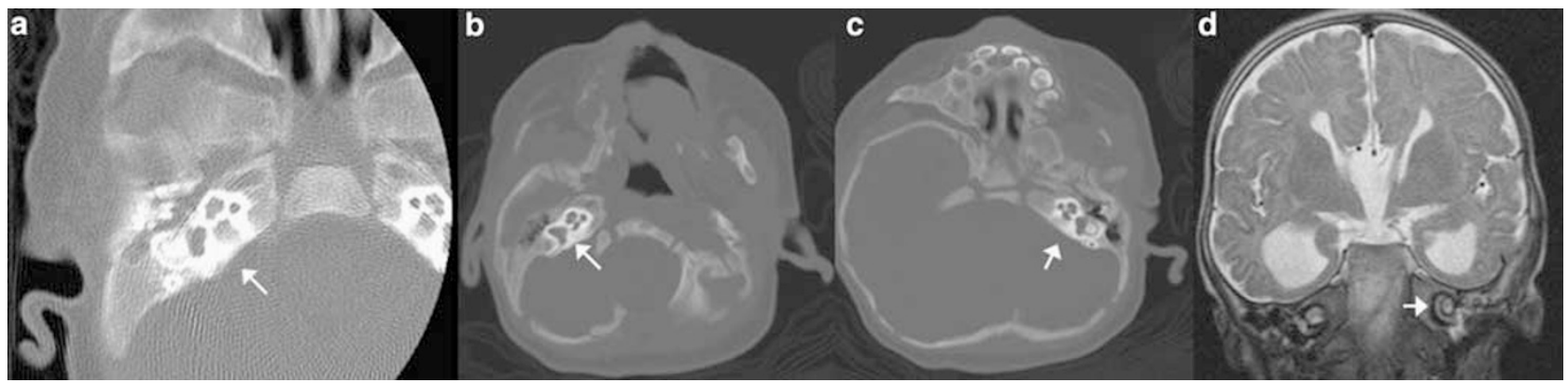

Figure 2 Imaging showing normal two and a half turns of the cochlea in patients 2 (a), 3 (b, c) and 4 (d). The normal cochlear anatomy implies that the hearing loss could be secondary to abnormal sensory epithelium or its connecting pathways. 
examination revealed esotropia of the left eye. She had global developmental delay; she sat unsupported at 1 year, crawled at 14 months and could not walk without support at 18 months (age at last evaluation). Her expressive language was delayed, and at 18 months, she did not have any words.

\section{Patient 3}

The proband was born to nonconsanguineous Hispanic parents at 39 weeks of gestation by Cesarean section performed for nonprogression of labor. Family history was noncontributory. Pregnancy was uncomplicated; however, prenatal ultrasounds revealed the possibility of hydrocephalus and agenesis of the corpus callosum (ACC). Growth parameters at birth for weight $(2.62 \mathrm{~kg}$, 3rd centile) and FOC $(32 \mathrm{~cm} ; 0.1$ centile, $z$-score of -3.0 ) were below normal, but the length $(48 \mathrm{~cm}, 5$ th centile $)$ was within normal limits. The perinatal period was complicated by feeding difficulties that mandated the placement of a feeding tube. Examination at 2 months of age revealed a FOC of $33 \mathrm{~cm}$ ( 0.1 centile, $z$-score of -5.0$)$, length of $52 \mathrm{~cm}(0.5$ centile, $z$-score -2.6$)$ and a weight of $3.45 \mathrm{~kg}$ (0.3 centile, $z$-score -2.7$)$. Dysmorphic features included low set and posteriorly rotated ears, midface hypoplasia, a high arched palate, bifid uvula, small hands with mild fifth finger clinodactyly and small feet. CT scan of the head at 2 months of age revealed the absence of corpus callosum with colpocephaly (Figure $3 \mathrm{~b}$ and c), a marked increase in the size of the lateral ventricles, and premature fusion of both lambdoid as well as the posterior aspect of the superior sagittal sutures giving rise to plagiocephaly. Auditory brainstem response (ABR) showed mild conductive loss in the left ear and a normal auditory acuity in the right ear. CT scan showed normal cochlear anatomy (Figure $2 \mathrm{~b}$ and $\mathrm{c}$ ). At the 10-month examination, her weight $(5.38 \mathrm{~kg}, 0.1$ centile, $z$-score -4.0$)$, length $(64 \mathrm{~cm}, 0.3$ centile, $Z$-score -2.7$)$ and FOC $(39.5,0.1$ centile, $z$-score -4.3 ) were well below the normal limits. She had significant delay and had not attained any motor or language milestones. Peripheral blood karyotyping performed elsewhere was reported as 46,XX,del(6)q25.2$\mathrm{q} 25.3$.

\section{Patient 4}

This male patient was born to a 30-year-old-gravida 2, para1-woman with no significant medical history. The parents are nonconsanguineous, and family history was noncontributory. Pregnancy was uncomplicated until 31 weeks of gestational age when prenatal ultrasound revealed enlarged cerebral ventricles. The proband was born through vaginal delivery at 38 weeks of gestation. The growth parameters were remarkable for a weight of $2.8 \mathrm{~kg}$ ( 3 rd centile), length of $47 \mathrm{~cm}$ ( 1.1 centile, $z$-score -2.3$)$ and an FOC of $31 \mathrm{~cm}$ ( 0.4 centile, $z$-score -2.7$)$. Apgar scores were 6 and 8 at 1 and $5 \mathrm{~min}$, respectively. The perinatal period was unremarkable. At the 2-month examination, his FOC of $34.5 \mathrm{~cm}(0.1$ centile, $z$-score -4.2$)$ and length of $53 \mathrm{~cm}$ ( 0.1 centile, $z$-score -3.0$)$ were well below the normal, whereas the weight $(4.44 \mathrm{~kg})$ was at the 3 rd centile. Dysmorphic features including mild midface hypoplasia, low set posteriorly rotated ears with abnormal helices and high arched palate were evident (Figure 1c). The hands were held in a clenched position, but there were no overlapping fingers and palmar creases were normal. The lower limbs had a mild valgus deformity at the knee and rocker bottom feet. There was mild hypertonia in all four extremities with normal deep tendon reflexes. He had penoscrotal webbing that was surgically corrected with scrotoplasty at 6 months. Ophthalmological examination was abnormal and showed intermittent esotropia, lash ptosis and strabismic amblyopia that was treated with eye patching. MRI of the brain showed agenesis of corpus callosum with accompanying colpocephaly and a diffuse delay in myelination (Figure 3a). ABR was consistent with a moderate-to-severe sensorineural hearing loss in both ears. The cochlear anatomy was unremarkable (Figure 2d). Echocardiogram showed a fenestrated septum secundum ASD with minimal left-to-right shunting. At 10 months, he continued to be microcephalic with an FOC of $40.4(0.1$ centile, $z$-score -4.5$)$, whereas his weight $(8 \mathrm{~kg})$ and length

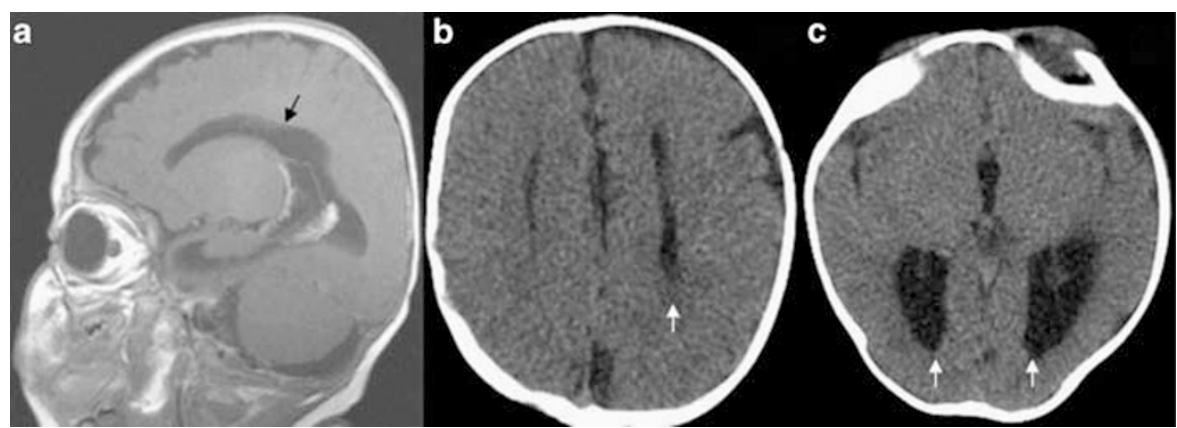

Figure 3 (a) MRI showing the absence of corpus callosum in patient 4. CT scan showing parallel orientation of lateral ventricles (b) and colpocephaly (c) suggestive of agenesis of corpus callosum in patient 3. 
$(69.5 \mathrm{~cm})$ were at the 5 th and 6 th centiles, respectively. He had global delay and could not sit, crawl or rollover. G-banded karyotyping performed on peripheral blood leukocytes was consistent with a terminal deletion of $6 \mathrm{q}$; 46XY,del(6)(q25.3).

\section{Material and methods Human subjects}

Following an initial result of the chromosome microarray analysis (CMA), ${ }^{10}$ informed consent was obtained from the parents for further analysis by whole-genome array-based oligonucleotide comparative genomic hybridization (CGH). The research protocol was approved by the Institutional Review Board (IRB) for Baylor College of Medicine and affiliated hospitals.

\section{Clinical chromosome microarray analysis}

CMA was initially performed on all four cases. The microarrays were designed and manufactured in the Medical Genetics Laboratory as described previously. ${ }^{10}$ The procedures for DNA digestion, labeling and hybridization, as well as data analysis were performed as described previously. ${ }^{11}$

\section{FISH analysis}

Confirmatory FISH analyses with the BAC clones were performed using standard procedures. Briefly, the BAC clone of interest was grown in broth media with $20 \mu \mathrm{g} / \mathrm{ml}$ of chloramphenicol. DNA was extracted from bacterial artificial chromosome clones (Eppendorf Plasmid Mini Prep kit, Hamburg, Germany) and directly labeled with SpectrumOrangeTM dUTP by nick-translation (Vysis, Downer Grove, IL, USA) according to the manufacturers' instructions.

\section{High-resolution oligonucleotide array}

The Agilent 244K Whole Human Genome Oligo Microarray Kit (Agilent Technologies Inc., Santa Clara, CA, USA) contains 238459 arrayed 60-mer oligonucleotides, representing a compiled view of the human genome at an average resolution of $6.4 \mathrm{~kb}$. The procedures for DNA digestion, labeling and hybridization were performed according to the manufacturer's instructions with some modifications. $^{12}$

\section{Results}

\section{Clinical features}

The clinical phenotype of the patients is shown in Table 1. Microcephaly, developmental delay and dysmorphic features, such as hypertelorism, abnormal root of the nose, midface hypoplasia and ear abnormalities were common to all (Figure 1). Plagiocephaly and growth restriction were seen in three of the four patients. Moderate-to-severe sensorineural loss was found in patients 1, 2 and 4, whereas patient 3 had mild conductive hearing loss. ACC was seen in patients 3 and 4 .

\section{Clinical chromosome microarray analysis}

We initially performed array-CGH (a-CGH) analysis on DNA extracted from blood leukocytes on the clinical microarray platform routinely used in our institution. The interrogating BAC clones deleted are shown in Table 2.

\section{High-resolution oligonucleotide array}

To determine the precise size extent and genomic content of the deletions, the patient samples were analyzed using an Agilent $244 \mathrm{~K}$ oligonucleotide array. The breakpoints are as depicted in Table 2. The deletions varied in size from 3.77 to $13.81 \mathrm{Mb}$ (Figure 4 ). The commonly deleted genomic interval (SRO) corresponds to chromosomal region 6q25.2-25.3 and encompasses an area of $3.52 \mathrm{Mb}$ that contains 15 genes mapping from TIAM2 (T-cell lymphoma invasion and metastasis 2) to TULP4 (Figure 5).

\section{Discussion}

Interstitial deletions of the long arm of chromosome 6 are rare. ${ }^{2-7,14,15}$ We report and characterize four patients with de novo interstitial deletions of $6 \mathrm{q}$, including two submicroscopic deletions. The deletions were of different sizes with the SRO $(3.52 \mathrm{Mb})$ corresponding to the chromosomal segment $6 \mathrm{q} 25.2-\mathrm{q} 25.3$.

The clinical features of our cases were compared with four other cases with similar interstitial deletions that had breakpoints determined cytogenetically to map between 6q24 and 6q27 and encompassing the SRO of our cases (Table 1). ${ }^{4,6,14,16}$ This approach may help further delineate the phenotype associated with the deletion of $6 q 25.2-q 25.3$. The studies with terminal deletions of $6 q$ with breakpoints at or distal to $6 \mathrm{q} 24.3$, the most proximal breakpoint of deletions in our cases, and containing the SRO seen in our cases are also summarized..$^{5,17-21}$ Studies with $6 \mathrm{q}$ monosomy because of translocations, deletions with proximal breakpoints centromeric to $6 \mathrm{q} 24$ or those not deleted for the 6q25.2-q25.3 were excluded from comparison.

Developmental delay in varying degrees was found in all the cases across all the above-mentioned studies. Growth restriction was found only in two of our patients (patients 3 and 4), whereas it was observed more commonly in patients with larger, cytogenetically visible deletions. This discrepancy may be because of the fact that the extent of deletions in the other studies may have been underestimated by the limited genomic resolution of G-banding analysis. Most of the dysmorphic features seen in our patients were nonspecific and have been reported in other studies as well (Table 1). The shared specific findings in our 
Table 1 Clinical features observed in our cohort compared to those seen in studies with similar cytogenetically determined deletions

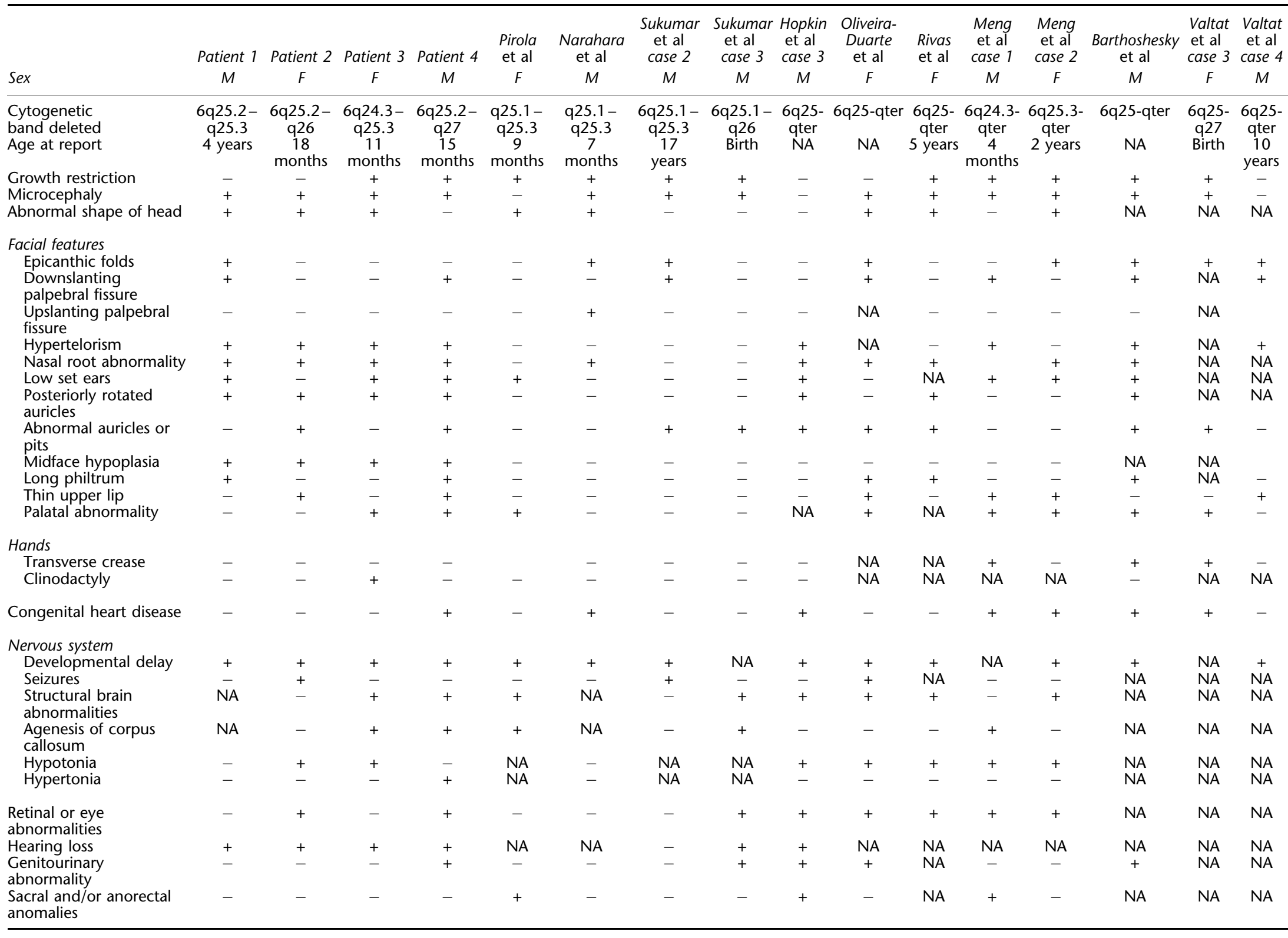

The first four studies involve interstitial deletions around the SRO seen in our cases, whereas the others involve terminal deletions encompassing the SRO. + Denotes that a characteristic is present, - denotes the absence of the characteristic and NA implies that data were not available. 
cases appear to be the association of microcephaly, ACC and hearing loss with deletion of the $6 \mathrm{q} 25$ region.

Microcephaly was present in all of our patients as well in all studies with interstitial or terminal deletions involving 6q25 except two cases. ${ }^{2,17}$ (Table 1). In the case of Hopkin et $a l^{2}{ }^{2}$ the proband had macrocephaly because of hydrocephalus, whereas in Valtat et al, ${ }^{17}$ the FOC was $1.5 \mathrm{SD}$ below the mean. Pirola et $\mathrm{al}^{16}$ reported a normal head circumference (50th centile) at 9 months of age in their case, but the birth FOC was 2 SD below the mean. ACC was seen in two of our patients ( 3 and 4 ), and has been reported in other 6q-deletion patients. ${ }^{5,6,15,16,22}$ By comparing these studies, we delimited a critical region of $3.3 \mathrm{Mb}$ for ACC (Figure 6). This interval falls within the SRO of $3.52 \mathrm{Mb}$ seen in our cases. However, there may be incomplete penetrance for this abnormality, as some cases with deletions of this critical region (eg, patient 2) have normal corpus callosal development. Hearing loss was seen in all of our patients. Hopkin $e t a l^{2}$ describe sensorineural hearing loss in one of their cases, whereas Sukumar et $\mathrm{al}^{6}$ mention that the hearing of one of the patients was abnormal, but do not provide results of objective testing. The other reports do not specifically mention the hearing evaluation. There are two known regions in the vicinity $6 \mathrm{q} 25$ that have been implicated in deafness. One locus at 6q26-q27 identified by linkage analysis in a consanguineous family is associated with autosomal recessive congenital sensorineural deafness, whereas the other region at $6 \mathrm{q} 23.2$ harbors the EYA4 gene implicated in later onset of autosomal dominant hearing loss. ${ }^{23,24}$

Our data suggest that microcephaly, ACC and hearing loss seen in interstitial or terminal deletions involving 6q25 may map to a 3.52-Mb region at 6q25.2-q25.3. This region harbors 12 protein-coding genes (Figure 5); among them, the ones that may be pertinent to the clinical phenotype are TIAM2, NOX3 and SYNJ2.

TIAM2 encodes a guanine nucleotide exchange factor. A highly similar protein (Stef) is implicated in normal neural development in a murine model. ${ }^{25}$ The pattern of expression of Stef in a stage and region-specific manner in the mouse brain corresponded to neuronal morphological changes.

SYNJ2 is a phosphoinositol 5-phosphatase that is involved in vesicular trafficking and actin dynamics. In glioblastoma cell lines, SYNJ2 is implicated in the regulation of the formation of invadopodia and lamellipodia leading to tumor cell invasion and migration. ${ }^{26}$ It is, however, not known whether SYNJ2 has a role in normal migration of neurons during development. It is tempting to hypothesize that TIAM2 and SYNJ2 may be involved in normal development of the brain and that haploinsufficieny of either or both causes microcephaly, ACC and developmental delay seen in 6q25.3 deletions.

NOX3 is an NADPH oxidase that is implicated in normal development of otoconia in mice. ${ }^{27}$ Homozygous mutations lead to lack of otoconia and significant vestibular 


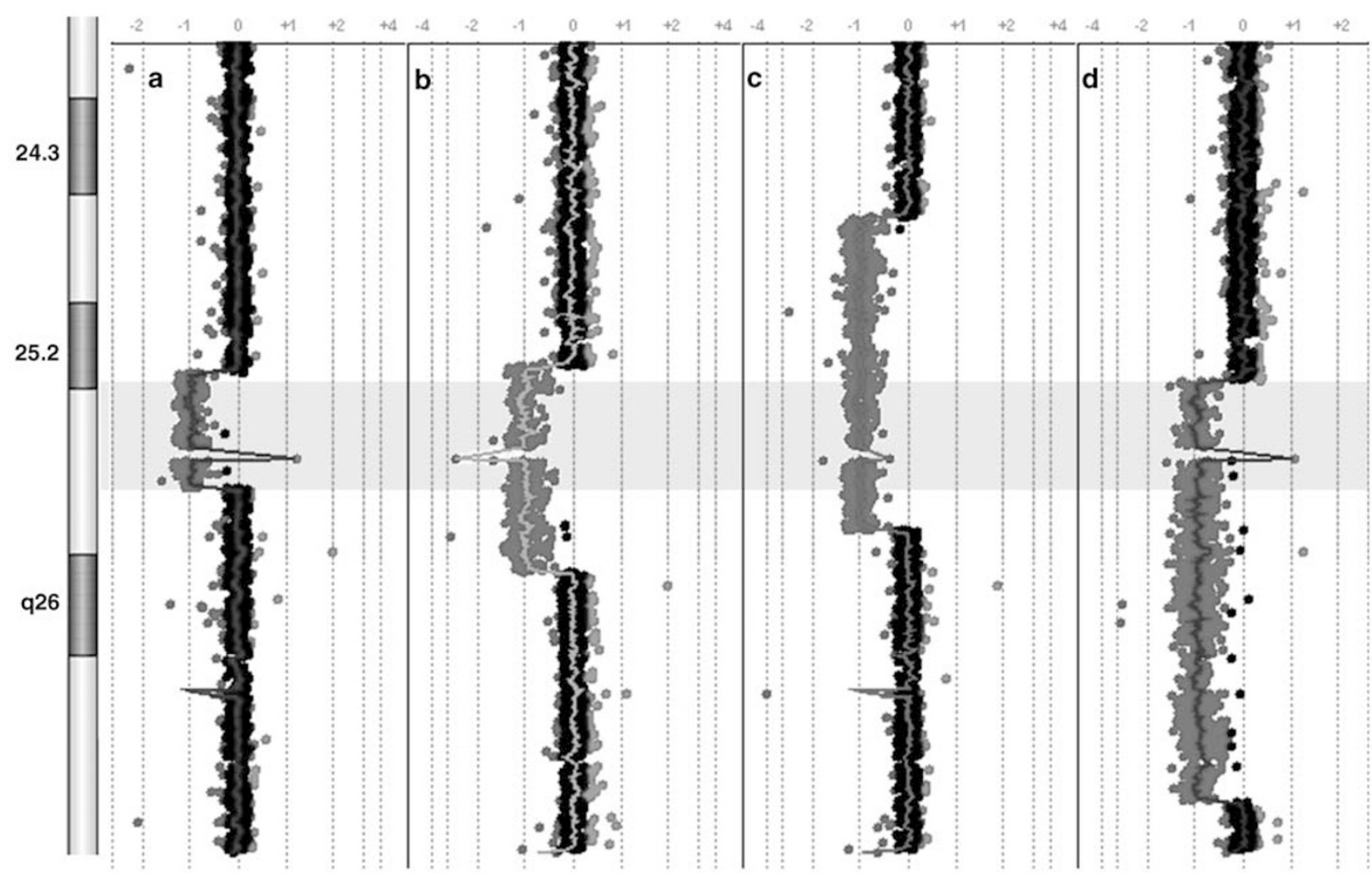

Figure 4 Mapping of breakpoints and common deleted region (blue; $\mathbf{a}, \mathbf{b}, \mathbf{c}$ and $\mathbf{d}$ ). Each point represents an oligonucleotide probe. The normalized data for each probe is represented along a vertical line that indicates its relative position on 6q. Loss of copy number is indicated by deviation to the left of the center (depicted in green), whereas gain of copy number is indicated by deviation to the right of the center (depicted in red). The deletions of four patients span $3.77,6.7,10.3$ and $13.81 \mathrm{Mb}$. The commonly deleted region (shaded) spans $3.52 \mathrm{Mb}$ from 155349955 to $158870729 \mathrm{bp}$. The spike in the $6 \mathrm{q} 25.3$ region represents the copy number variation (CNV) of the only one oligonucleotide (60 mer, positioned at $157888702-157888761 \mathrm{bp}$ ) in the flanking $420-\mathrm{Kb}$ genomic region. The oligonucleotide is located in a known CNV region and is most likely indicative of polymorphism. Note that there is grouping of proximal breakpoints in patients 1, 2 and 4 . A full-colour version of this figure is available at the EJHG journal online.

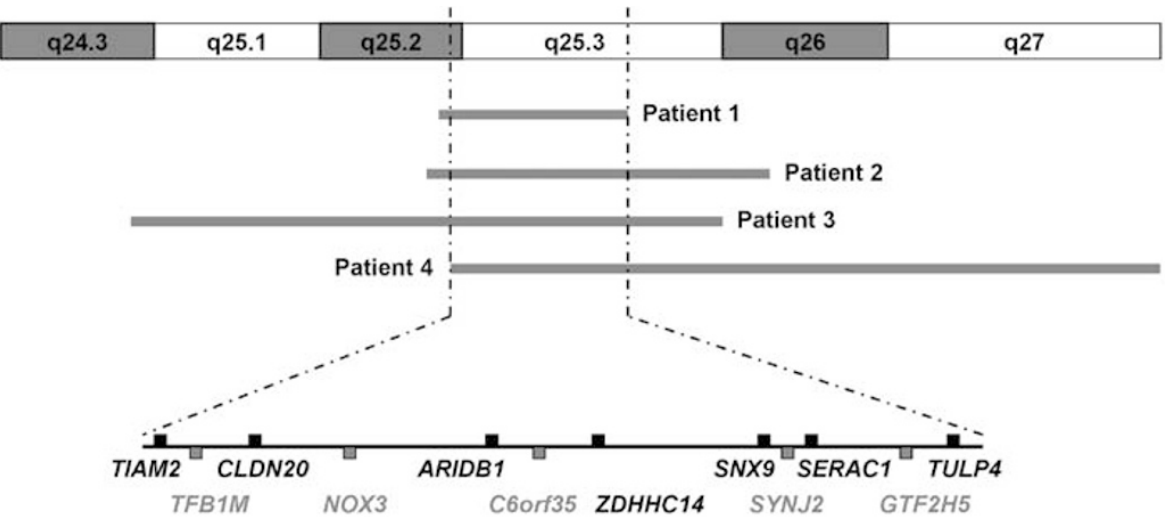

Figure 5 Protein-coding genes in the smallest region of overlap (SRO). All the genes except NOX3 are expressed in the brain.

dysfunction, whereas the heterozygous mice had normal auditory and vestibular functions. The other inner ear structures including the sensory epithelium and the hearing of the affected mice were normal. It is, however, possible that haploinsufficieny of NOX3 may have auditory as well as vestibular effects in humans. 


\begin{tabular}{|c|c|c|c|c|c|}
\hline $6 q 24.3$ & $6 q 25.1$ & $6 q 25.2$ & $6 q 25.3$ & $6 q 26$ & $6 q 27$ \\
\hline & & & & & Patient 3 \\
\hline & & & & & Patient 4 \\
\hline & & & & & Pirola et al \\
\hline & & & & & Sukumar et al \\
\hline & & & & & Meng et al \\
\hline
\end{tabular}

Figure 6 The distal deletions of $6 q$ associated with agenesis of the corpus callosum (ACC) share a common minimal region (gray) that is within the smallest region of overlap (SRO) seen in our cases (red bars). This indicates that a gene important for normal corpus callosal development lies in the $6 \mathrm{q} 25.2-\mathrm{q} 25.3$ region. A full-colour version of this figure is available at the EJHG journal online.

Genomic rearrangements describe mutational changes in the genome such as duplication, deletion, insertion and inversion that are different from the traditional WatsonCrick base pair alterations. Nonrecurrent genomic rearrangements can be mediated by nonhomologous end joining $(\mathrm{NHEJ})^{28}$ or fork stalling and template switching (FoSTeS). ${ }^{29}$ Given that there was grouping of the proximal breakpoints in three of our patients (Figure 4), it is possible that an underlying genomic architecture important to the rearrangement is present in the vicinity of the deleted regions.

In summary, interstitial deletions involving 6q25.2q25.3 described herein presented with microcephaly, ACC, developmental delay, hearing loss and dysmorphic features. The deletions can remain undetected by routine karyotyping techniques as evidenced by normal karyotyping results in two of our patients. We hence recommend a-CGH for diagnosis of such deletions. From a clinical standpoint, we suggest that MRI of the brain and a formal hearing evaluation should be considered in all patients with deletions involving $6 \mathrm{q} 25$.

\section{Acknowledgements}

We thank the participating families for their kind cooperation. This work was supported in part by fellowship grants from the Osteogenesis Imperfecta Foundation (SNSC) and the National Urea Cycle Foundation and Children's National Medical Center (AE).

\section{References}

1 Milosevic J, Kalicanin P: Long arm deletion of chromosome no. 6 in a mentally retarded boy with multiple physical malformations. J Ment Defic Res 1975; 19: 139-144.

2 Hopkin RJ, Schorry E, Bofinger $\mathrm{M}$ et al: New insights into the phenotypes of 6q deletions. Am J Med Genet 1997; 70: 377-386.

3 Matkins SV, Meyer JE, Berry AC: A child with partial monosomy $6 \mathrm{q}$ secondary to a maternal direct insertional event. J Med Genet 1987; 24: 227-229.

4 McLeod DR, Fowlow SB, Robertson A, Samcoe D, Burgess I, Hoo JJ: Chromosome 6q deletions: a report of two additional cases and a review of the literature. Am J Med Genet 1990; 35: 79-84.

5 Meng J, Fujita H, Nagahara N, Kashiwai A, Yoshioka Y, Funato M: Two patients with chromosome $6 \mathrm{q}$ terminal deletions with breakpoints at q24.3 and q25.3. Am J Med Genet 1992; 43: 747-750.

6 Sukumar S, Wang S, Hoang K et al: Subtle overlapping deletions in the terminal region of chromosome 6q24.2-q26: three cases studied using FISH. Am J Med Genet 1999; 87: 17-22.

7 Titomanlio L, Giurgea I, Baumann C et al: A locus for sacral/ anorectal malformations maps to $6 \mathrm{q} 25.3$ in a $0.3 \mathrm{Mb}$ interval region. Eur J Hum Genet 2006; 14: 971-974.

8 Pandya A, Braverman N, Pyeritz RE, Ying KL, Kline AD, Falk RE: Interstitial deletion of the long arm of chromosome 6 associated with unusual limb anomalies: report of two new patients and review of the literature. Am J Med Genet 1995; 59: 38-43.

9 Schuster M, Lohscheller J, Kummer P, Eysholdt U, Rosanowski F: Severe sensory hearing loss in $\operatorname{del}(6 \mathrm{q})$-syndrome. Int I Pediatr Otorhinolaryngol 2003; 67: 1263-1266.

10 Cheung SW, Shaw CA, Yu W et al: Development and validation of a CGH microarray for clinical cytogenetic diagnosis. Genet Med 2005; 7: 422-432.

$11 \mathrm{Lu} \mathrm{X}$, Shaw CA, Patel A et al: Clinical implementation of chromosomal microarray analysis: summary of 2513 postnatal cases. PLOS ONE 2007; 2: e327.

12 Probst FJ, Roeder ER, Enciso VB et al: Chromosomal microarray analysis (CMA) detects a large $\mathrm{X}$ chromosome deletion including FMR1, FMR2, and IDS in a female patient with mental retardation. Am J Med Genet A 2007; 143: 1358-1365.

$13 \mathrm{Ou}$ Z, Kang SH, Shaw CA et al: Bacterial artificial chromosomeemulation oligonucleotide arrays for targeted clinical arraycomparative genomic hybridization analyses. Genet Med 2008; 10: $278-289$.

14 Narahara K, Tsuji K, Yokoyama Y et al: Specification of small distal $6 \mathrm{q}$ deletions in two patients by gene dosage and in situ hybridization study of plasminogen and alpha-L-fucosidase 2 . Am J Med Genet 1991; 40: 348-353.

15 Rubtsov N, Senger G, Kuzcera H et al: Interstitial deletion of chromosome 6q: precise definition of the breakpoints by microdissection, DNA amplification, and reverse painting. Hum Genet 1996; 97: 705-709.

16 Pirola B, Bortotto L, Giglio S et al: Agenesis of the corpus callosum with Probst bundles owing to haploinsufficiency for a gene in an $8 \mathrm{cM}$ region of 6q25. J Med Genet 1998; 35: 1031-1033.

17 Valtat C, Galliano D, Mettey R, Toutain A, Moraine C: Monosomy 6q: report on four new cases. Clin Genet 1992; 41: 159-166.

18 Oliveira-Duarte MH, Martelli-Soares LR, Sarquis-Cintra T, Machado ML, Lison MP: Distal monosomy of the long arm of chromosome 6 (6q25-6qter) inherited by maternal translocation $\mathrm{t}(6 \mathrm{q} ; 17 \mathrm{q})$. Ann Genet 1990; 33: 56-59.

19 Rivas F, Ruiz C, Rivera H, Moller M, Serrano-Lucas JI, Cantu JM: De novo del(6)(q25) associated with macular degeneration. Ann Genet 1986; 29: 42-44.

20 Bartoshesky L, Lewis MB, Pashayan HM: Developmental abnormalities associated with long arm deletion of chromosome No. 6 . Clin Genet 1978; 13: 68-71. 
21 Stevens CA, Fineman RM, Breg WR, Silken AB: Report of two cases of distal deletion of the long arm of chromosome 6. Am J Med Genet 1988; 29: 807-814.

22 Shen-Schwarz S, Hill LM, Surti U, Marchese S: Deletion of terminal portion of $6 \mathrm{q}$ : report of a case with unusual malformations. Am J Med Genet 1989; 32: 81-86.

23 Ansar M, Ramzan M, Pham TL et al: Localization of a novel autosomal recessive non-syndromic hearing impairment locus (DFNB38) to 6q26-q27 in a consanguineous kindred from Pakistan. Hum Hered 2003; 55: 71-74.

24 Hildebrand MS, Coman D, Yang T et al: A novel splice site mutation in EYA4 causes DFNA10 hearing loss. Am J Med Genet A 2007; 143A: 1599-1604.

25 Yoshizawa M, Hoshino M, Sone M, Nabeshima Y: Expression of stef, an activator of Rac1, correlates with the stages of neuronal morphological development in the mouse brain. Mech Dev 2002; 113: $65-68$.

26 Chuang YY, Tran NL, Rusk N, Nakada M, Berens ME, Symons M Role of synaptojanin 2 in glioma cell migration and invasion. Cancer Res 2004; 64: 8271-8275.

27 Paffenholz R, Bergstrom RA, Pasutto F et al: Vestibular defects in head-tilt mice result from mutations in Nox3, encoding an NADPH oxidase. Genes Dev 2004; 18: 486-491.

28 Lupski JR, Stankiewicz P: Genomic disorders: molecular mechanisms for rearrangements and conveyed phenotypes. PLoS Genet 2005; 1: e49.

29 Lee JA, Carvalho CM, Lupski JR: A DNA replication mechanism for generating nonrecurrent rearrangements associated with genomic disorders. Cell 2007; 131: 1235-1247. 